\title{
Spectral Measurement Techniques in Planetary Radar
}

\author{
G. Pettengill \\ Arecibo Ionospheric Observatory,* Arecibo, Puerto Rico
}

(Received December 27, 1963)

\begin{abstract}
The precise measurement of Doppler shift and dispersion (frequency broadening) of a radio echo returned from a planetary surface can yield useful information on planetary orbits and surface characteristics. Methods employed to measure Doppler displacements include banks of narrow receiving filters, phase-locked oscillator loops, and digital sampling and calculational techniques. The latter, while complex and sometimes slower, offer greater flexibility and in some cases enable measurements not otherwise possible.
\end{abstract}

\section{Introduction}

As the sensitivity of radar systems has grown in recent years through higher power transmitters, larger antennas, and low-noise receivers, so has the sophistication of the assigned tasks and measurement techniques. In the beginning it was sufficient merely to establish the presence of an echo with reasonable certainty. As stronger signals were obtained, precise measurements of time delay enabled accurate determinations of distance to the echoing body. With even stronger signals it was possible to see the degree to which the returned power was dispersed in time, and thus to study the radio scattering law of the distant surface.

The use of transmitters controlled by extremely stable frequency sources has also permitted the exploration of the frequency shift and dispersion of the returned signal. This coordinate, which is in a sense conjugate to the time dimension associated with radar range, contains useful information on the state of motion of the echoing body. In particular, if the frequency dispersion occasioned by surface roughness and rotation is small enough, it is possible to make measurements of the relative orbital motions of the earth and distant object with an accuracy which exceeds $0.1 \mathrm{~m} / \mathrm{sec}$ (see remarks relating to figures 3 and 4). Such precision can significantly improve the accuracy with which the orbits of the planets are known.

The frequency dispersion of the returned echo depends on the degree of surface roughness and the amount of rotation of the target. In some cases where the rotation is known from optical observations, or where the signal is sufficiently strong to permit unambiguous determination of the spectral limits corresponding to the limbs, the surface roughness may be deduced solely from the shape of the spectrum. Where this is not possible, an independent

\footnotetext{
* Operated by Cornell Unlversity for A RPA.
}

measure of surface roughness from the observed time dispersion may conversely be combined with the spectral data to yield a value for rotation.

An even more powerful method for studying the detailed scattering characteristics of a distant surface involves obtaining a frequency spectrum at each of a number of values of time delay. In this way it is possible to develop a map of surface reflectivity with detail which is partly determined by the frequency resolution available.

It is clear, therefore, that many of the objectives of radar astronomy are dependent on measuring accurately the amount by which various portions of the returned echo power have been displaced in frequency. An attempt will be made in the following section to describe some of the techniques which have been used.

\section{Techniques}

The techniques which have been used for spectral analysis may in general be broken down into those which are primarily analog and those which depend heavily on digital processing. The choice of a proper mix between the two for a given experiment will depend upon the echoing characteristics of the target and upon the speed and accuracy of the components available. Perhaps the most obvious technique is simply to use one or more very narrow-band filters to sequentially analyze portions of the received spectrum, as was done by Fricker et al. [1958], for lunar echoes. In this case a single $1 \mathrm{c} / \mathrm{s}$ filter was used, and the narrow-band tape-recorded data were rerun many times at different speeds until a complete spectrum of the desired region was obtained. The resolution of this system was limited by its stability to about $1 \mathrm{c} / \mathrm{s}$.

In another approach to the problem used by Browne et al. [1956], the autocorrelation function for the temporal fading of the received power was calcu- 
lated. By taking the Fourier transform of the measured autocorrelation function, these workers were able to obtain a power spectrum of the received signal, without the stability restrictions imposed by direct analog filtering. Since they worked with a detected signal, however, their spectrum was folded and suffered some loss of information.

More recently, Goldstein [1961] and Weinreb [1963] have cleverly adapted the autocorrelation approach to spectral analysis to digital manipulation. By severely limiting the amplitude excursion of the received signal to a point where it could be described as simply positive or negative (one-bit quantization) the calculation of the autocorrelation function becomes very rapid. Both workers analyzed bandwidths of about $35 \mathrm{kc} / \mathrm{s}$ sampled at about 70 $\mathrm{kc} / \mathrm{s}$ using simple logical circuits and delay lines to calculate between 20 and 45 channels of delay. Calculation was rapid enough to permit the data to be processed and integrated as rapidly as it was received. Necessary weighting and Fourier transformation (see eq (4)) of the calculated autocorrelation function was performed in a few minutes at the end of the run.

The advantages of this novel technique of spectral analysis are great while the drawbacks are small. The advantages consist in the high degree of stability afforded by a digital system which in effect synthesizes a large number of identical filters. Integration can proceed over a number of days in order to improve the statistics of a noisy signal, while the precision with which the various analyzing filters can be synthesized enables one to detect very small differences between adjacent portions of the spectrum. The drawback lies chiefly in the decreased final-output signal-to-noise ratio produced by the amplitude clipping. If the processed signal has Gaussian statistics, however, this loss may be made up completely by merely extending the observing period by a factor of approximately two. A block diagram of a typical system of this sort is shown in figure 1.

The processing may be described mathematically as follows [Weinreb, 1963]. Let $X(t)$ be the voltage waveform at some convenient (band-limited) intermediate frequency. Define:

$$
\begin{aligned}
& Y(t)=1, \text { when } X(t)>0 ; \\
& Y(t)=-1, \quad X(t)<0 .
\end{aligned}
$$

Sample to obtain $Y(t)$ at intervals: $\Delta t=1 / 2 B$, where $B=$ bandwidth of signal. Calculate $N$ values (channels) of delay for the one-bit autocorrelation at multiples of $\Delta t$ :

$$
\rho_{n}^{\prime}(n \Delta t) \frac{1}{K} \sum_{k=1}^{K} y(k \Delta t) y(k \Delta t+n \Delta t)
$$

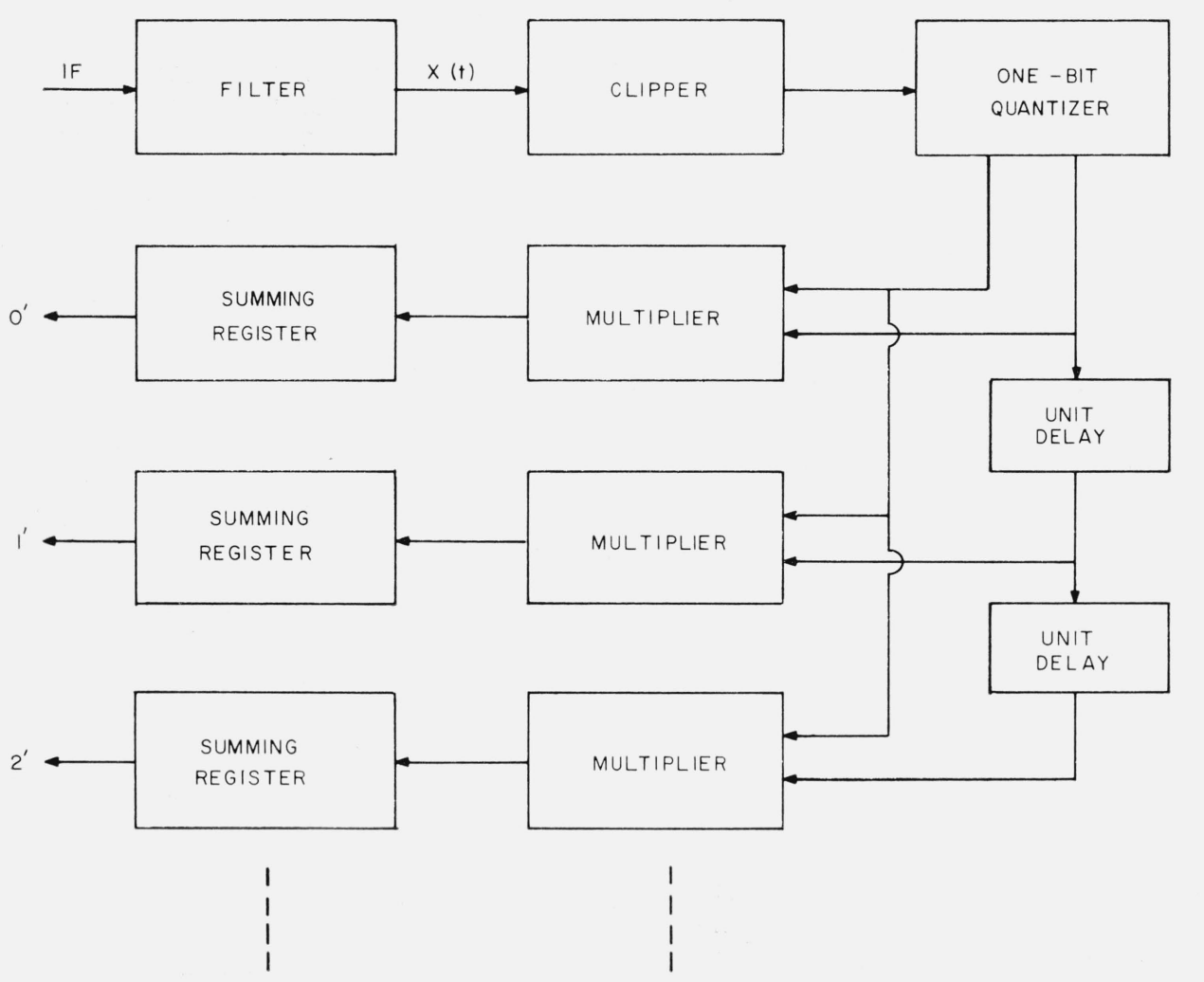

Figure 1. Block diagram of the one-bit autocorrelation system. 
where the integration in any channel has been allowed to proceed for a time $T=K \Delta t$. Correct the resulting values of the one-bit autocorrelation function according to the relation of VanVleck [1943]:

$$
\rho_{n}(n \Delta t)=\sin \left[(\pi / 2) \rho_{n}^{\prime}(n \Delta t)\right]
$$

the spectral estimate is then found from the Fourier transform of the autocorrelation:

$$
P(f)=2 \Delta t\left[\rho_{0}+\sum_{n=1}^{N-1} \rho_{n} W_{n} \cos (2 \pi f n \Delta t)\right],
$$

where $W_{n}$ is a weighting function chosen so that $W_{0}=1$ and $W_{n}=0$ when $n>N-1$. The value of $N$ and the values of $W_{n}$ for $0<n<N-1$ are chosen according to the desired frequency resolution and sidelobe level; generally, the greater the resolution, the greater will be the fluctuation in the spectral estimate for a given amount of observed data [Blackman and Tukey, 1958]. The resulting spectral estimate will be normalized; it is a consequence of the one-bit quantization that absolute signal level is not preserved.

A somewhat different approach has been used by Pettengill and Henry [1960, 1962] to study the moon. In this method a series of unnormalized spectra is desired of a nongaussian signal. The spectra have been calculated directly from digitalized samples of two waveforms representing the sine and cosine components of the returned echo signal.
Amplitude clipping was negligible since 9-bit quantization (512 levels) was used to sample each component. In this measurement the incoming signal represented the echo of a short radar pulse, rather than a continuously received waveform as in the earlier cases. Thus the sampling interval is set by the interpulse interval of the radar, which in the experiments described above was about 40 msec. Such a long sampling interval was possible because the returned spectrum was never wider than about $10 \mathrm{c} / \mathrm{s}$, while the sampling rate permitted an unambiguous measurement over a window of $25 \mathrm{c} / \mathrm{s}$.

Figure 2 shows a block diagram of the setup. Fourier transformation was carried out over 20 individual four second segments of the received signal in a digital computer associated with the radar. The squared magnitudes of the transforms were then summed to yield the spectral estimate. (This corresponds to a linear taper on the $W_{n}$ in (4)). Because of the length of the digital word, it was not possible to carry out the spectral processing as the data were taken. Records were made on either paper or magnetic tape and reduced at a later time. The frequency stability of the equipment was better than $0.01 \mathrm{c} / \mathrm{s}$ over several minutes of time, permitting useful resolutions as low as $0.1 \mathrm{c} / \mathrm{s}$ and a doppler measurement accuracy approaching $0.01 \mathrm{c} / \mathrm{s}$. Figures 3 and 4 show sample echo spectra taken from the Moon and Venus, respectively. The resolution is about $0.25 \mathrm{c} / \mathrm{s}$, and the theoretical standard deviation of the fluctuation is 22 percent of the mean value of the estimate at each frequency. (This corresponds to 40 Blackman-Tukey degrees of free-

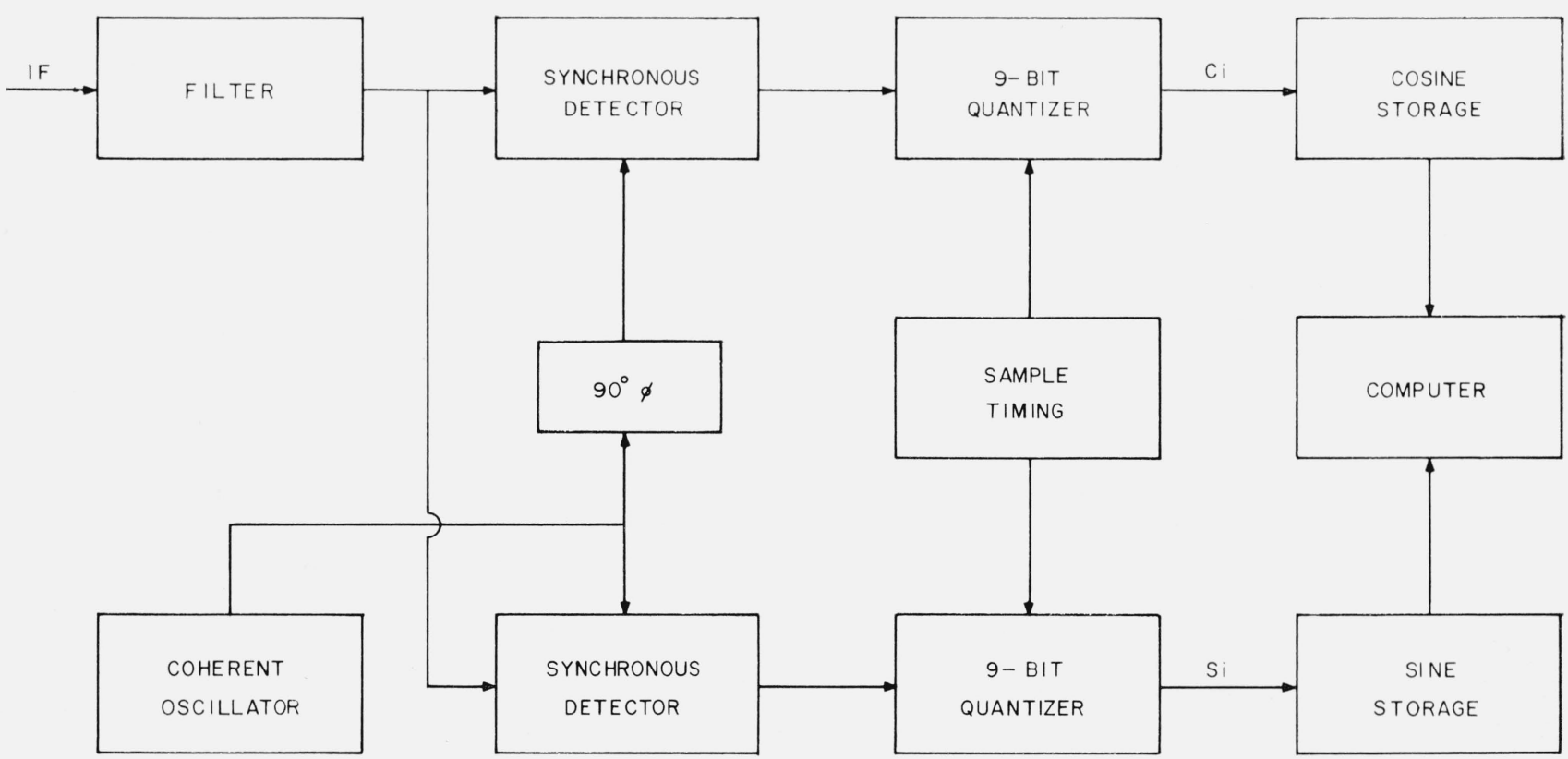

Figure 2. Block diagram of the 9-bit digital system used to obtain power spectra. 

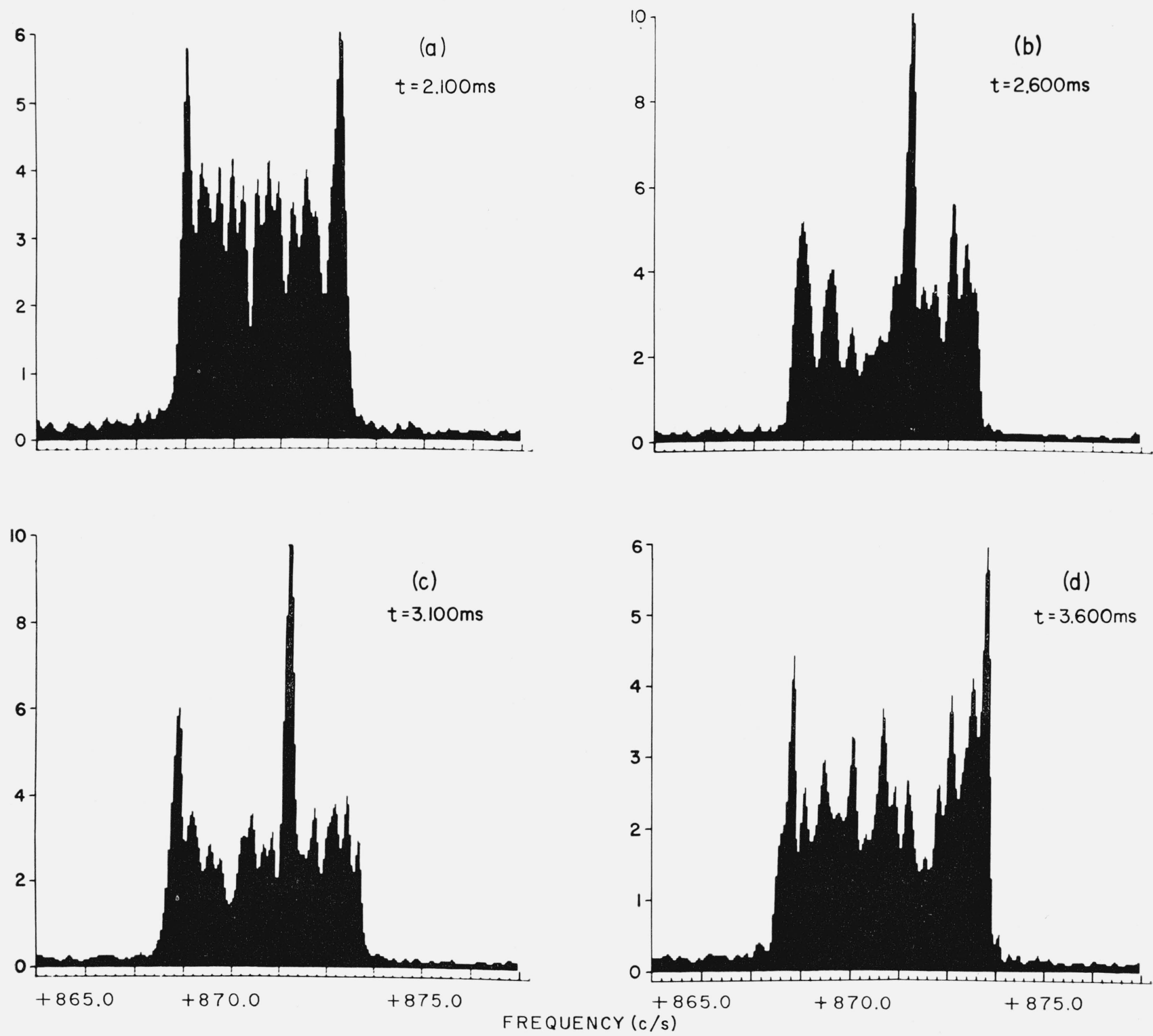

FIguRE 3. Lunar echo power spectra at 4 intervals of range taken by Millstone Radar of MIT at 19 h 00m $19 \mathrm{~s}$ UT, June $20,1961$.

dom.) Note that although the spectra repeat at multiples of the sampling frequency, the correct region can be selected easily from a priori knowledge. In the cases shown it is quite easy to locate the spectral center to within $0.1 \mathrm{c} / \mathrm{s}$; since the operating frequency was $440 \mathrm{Mc} / \mathrm{s}$, the radial component of the relative orbital motion of the Moon or Venus can be determined to better than $0.1 \mathrm{~m} / \mathrm{sec}$.

As Weinreb [1963] has shown, there is very little difference in digital processing time between the direct and autocorrelation routes to a power spec- trum when clipping is not permitted. What difference there is, however, favors the autocorrelation function as a useful intermediary around which to reduce the fluctuation statistics of the signal by integration.

One of the first to apply coherent digital processing to planetary echoes was Price [1959]. In this first realization, 6-bit samples were taken of a filtered IF signal at $15 \mathrm{kc} / \mathrm{s}$ and stored on magnetic tape. Although permitting great flexibility in searching out a signal of unknown bandwidth and location in 


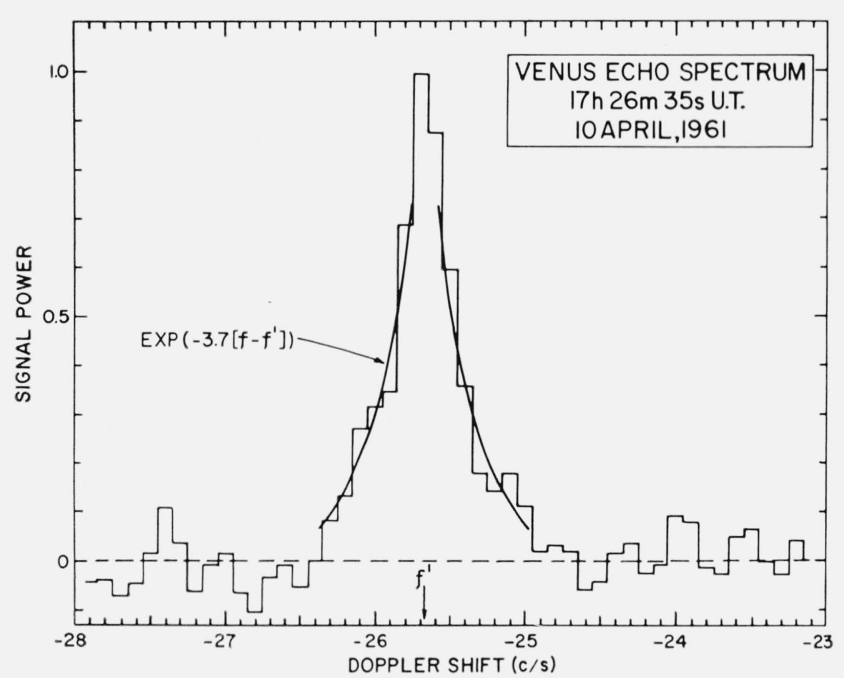

Figure 4. Venus echo spectrum obtained by Millstone Radar of MIT at time of inferior conjunction, 10 April 1961. Analyzing slit width used was $0.2 \mathrm{c} / \mathrm{s}$.

The smooth curve represents an exponential approximation to the observar tions. Transmitter frequency was $440 \mathrm{Mc} / \mathrm{s}$.

range and doppler frequency, this particular system required large quantities of computer time and would not be well matched to a continuing program.

We return finally to an analog method which has proven useful in planetary radar. Brockman [1961] describes an application of a phase-locked receiver to the problem of measuring precisely the doppler shift of Venus echoes. The use of receivers which phase lock to an incoming signal and maintain long integration times in a feedback loop has been commonplace in telemetry applications for many years. As applied to the detection of weak signals from Venus (and Mercury) this method permits accurate determination of Doppler shifts of continuous wave echoes with a minimum of processing complexity. In practice it is limited to those cases where the returned Doppler spectrum is quite narrow, say a few tens of cycle per second at the most. Because it tends to follow the center of intensity of the spectrum rather than the midpoint of the extremes, this method is probably less accurate than measurements made from a full spectrum. Nevertheless, in many applications its simplicity will make it attractive.

\section{Conclusion}

A number of spectral processing techniques have been described which range from fully analog to largely digital. Two avenues of approach to obtaining a power spectrum of a weak or fluctuating signal are available, one direct and one via the autocorrelation function. The proper mix of these techniques in any given application will depend on the signal characteristics and on the objectives of the experiment. It seems clear, however, that as faster digital computers become available at reasonable prices, and as greater stability and more sophisticated objectives are sought, the digital techniques will become more commonly employed.

\section{References}

Blackman, R. B., and J. W. Tukey (1958), The measurement of power spectra (Dover Publications, New York, N.Y.).

Brockman, M. H. (1961), Radar exploration of Venus, ed. Victor, Stevens, and Golomb, Jet Propulsion Laboratory Tech. Report No. 32-132, ch. V.

Browne, I, C., J. V. Evans, J. K. Hargreaves, and W. A. S. Murray (1956), Radio echoes from the moon, Proc. Phys. Soc. B 69, 901-290.

Fricker, S. J., R. P. Ingalls, W. C. Mason, M. L. Stone, and D. W. Swift (1958), Characteristies of moon-reflected UHF signals, MIT Lincoln Laboratory Tech. Report No. 187 (ASTIA 204,519).

Goldstein, R. M. (1961), Radar exploration of Venus, ed. Victor, Stevens, and Golomb, Jet Propulsion Laboratory Tech. Report No. 32-132, ch. IV.

Pettengill, G. H. (1960), Measurements of lunar reflectivity using the millstone radar, Proc. IRE 48, No. 5, 933, 934.

Pettengill, G. H., and J. C. Henry (1962), Enhancement of radar reflectivity associated with the lunar crater Tycho, J. Geophys. Res. 67, No. 12, 4881-4885.

Price, R. (1959), The venus radar experiment, paper presented at the Ninth General Assembly of AGARD, Aachen, Germany, 21 Sept. 1959.

Van Vleck, J. H. (1943), The spectrum of clipped noise, Harvard Radio Research Laboratory Report No. 51, 21 July 1943.

Weinreb, S. (1963), A digital spectral analysis technique and its application to radio astronomy, Ph. D. Thesis issued as MIT Research Laboratory of Electronics Tech. Report No. 412, 30 Aug. 1963. 